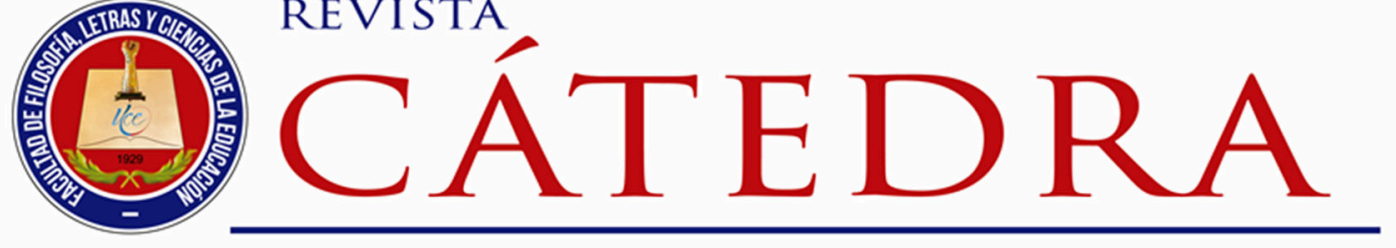

\title{
Análisis relacional del perfil de egreso del bachillerato general unificado y la oferta académica de la carrera de Pedagogía en Ciencias Experimentales Informática
}

Relational analysis of the graduation profile of the unified general baccalaureate and the academic offer of the Pedagogy in Experimental Sciences and Computer Science degree program

Gladys Cabascango-Trávez Universidad Central del Ecuador, Quito, Ecuador gmcabascango@uce.edu.ec https://orcid.org/0000-0002-8985-4971

Omar Pérez-Narváez

Universidad Central del Ecuador, Quito, Ecuador hperez@uce.edu.ec https://orcid.org/0000-0002-4496-2549

Javier Guaña-Moya Instituto Superior Tecnológico Japón, Quito, Ecuador eguana@itsjapon.edu.ec https://orcid.org/0000-0003-4296-0299

Nelson Salgado-Reyes Instituto Superior Tecnológico Japón, Quito, Ecuador nsalgado@itsjapon.edu.ec https://orcid.org/0000-0001-8908-7613

(Recibido: 14/11/2021; Aceptado: 18/11/2021; Versión final recibida: 22/12/2021)

Cita del artículo: Cabascango-Trávez, G., Pérez-Narváez, O., Guaña-Moya, J. y Salgado-Reyes, N. (2022). Análisis relacional del perfil de egreso del bachillerato general unificado y la 
oferta académica de la carrera de Pedagogía en Ciencias Experimentales Informática. Revista Cátedra, 5(1), 119-130.

\section{Resumen}

En el transcurso de la secundaria los estudiantes ecuatorianos se presentan al examen Ser Bachiller, componente que permite el acceso a la educación superior, es por ello que la presente investigación se enfocó en conocer los factores que inciden en la relación del perfil de egreso de los estudiantes de Bachillerato General Unificado (BGU) para el ingreso a la Carrera de Pedagogía en Ciencias Experimentales de la Informática de la Facultad de Filosofía Letras y Ciencias de la Educación en la Universidad Central del Ecuador. Se ejecutó una investigación no experimental, con enfoque de campo, de nivel descriptivo mediante la metodología cuantitativa se tomó datos; además se realizó un test, mismo que trabajó con los estudiantes que ingresan a la carrera como informantes esenciales y se procesó datos mediante el software estadístico SPSS 23. Entre los principales hallazgos se evidenció que los estudiantes graduados bajo el programa de estudio del BGU evidencia ciertas falencias ante las exigencias del perfil de ingreso que requiere la Universidad Central del Ecuador, además que las habilidades y destrezas adquiridas por los estudiantes del BGU son elementales, y las expectativas que los estudiantes alcancen el nivel de logro excelente es escaso; disminuyendo las posibilidades de ingreso a la educación superior.

\section{Palabras clave}

Bachillerato general unificado, informática, oferta académica, perfil de ingreso.

\section{Abstract}

In the course of high school, Ecuadorian students take the exam Ser bachiller, a component that allows access to higher education, that is why this research focused on knowing the factors that affect the relationship of the graduation profile of the students of the Unified General Baccalaureate for the entrance to the Pedagogy Career in Experimental Sciences of Computer Science of the Faculty of Philosophy, Letters and Educational Sciences at the Central University of Ecuador. Non-experimental research was carried out, with a field approach, at a descriptive level, using quantitative methodology, data was collected; in addition, a test was conducted, which worked with the students entering the career as essential informants and data was processed using SPSS 23 statistical software. Among the main findings, it was evidenced that the students graduated under the BGU program of study evidence certain deficiencies before the demands of the entrance profile required by the Central University of Ecuador, also that the skills and abilities acquired by the BGU students are elementary, and the expectations that the students reach the level of excellent achievement is scarce; decreasing the possibilities of entering higher education.

\section{Keywords}

Unified general baccalaureate, computer science, academic offerings, entrance profile.

\section{Introducción}

Acceder a la educación es un derecho en Ecuador (Constitución de la República del Ecuador (2008), y ofrecer una educación de calidad con competencias para el ámbito laboral y desarrollo personal es prioridad para las Organización de las Naciones Unidas y la UNESCO. En este sentido, el país ha establecido políticas de estado que son necesarias en la actualidad y que fomenten una mejor educación competitiva, sin embargo, la falta de competencias que presentan los estudiantes en nivel secundario son por el error en el sistema educativo, tal y como asevera (Terán et al., 2016, p.42). 
En la búsqueda de la calidad educativa, el país ha equivocado sus objetivos sobrecargando al docente de trabajo mayoritariamente en el aspecto administrativo sobre las necesidades del estudiante, haciendo que este se enfoque en la necesidad de cumplir con el trabajo que solicitan las autoridades, dejando de lado lo primordial que es la educación orientada a los estudiantes; manteniéndose un bachillerato general unificado, que evidencia absolutamente lo contrario a lo que se esperaba. Tal es el punto que, si en el 2017 Ecuador participó en las pruebas PISA D intentando alcanzar la media de las pruebas, en el 2018 según el director del Instituto Nacional de Evaluación Educativa (INEVAL) se decidió no participar y esperar a tal vez poder participar en las pruebas del 2024 (Rosero, 2019).

Alineado a lo anterior se hace un llamado a realizar los cambios necesarios en la educación secundaria que necesita replantear las asignaturas y el contenido del Bachillerato General Unificado (BGU), además el tiempo que se les asigna; pues no es cuanto abarca una asignatura sino la calidad de la misma y sobre todo que los conocimientos sean acertados por el estudiante. Ecuador al ser un país del tercer mundo enfrenta problemas en educación desde varios frentes el primero por la segregación social, en las zonas urbanas versus las zonas rurales. En las zonas urbanas se tiene diferentes limitantes como el acceso a ciertos recursos como lo son computadores, internet, laboratorios y aún a pesar de estar en una zona donde se cree que no debería existir carencias; aún persisten este tipo de limitantes, pero la mayoría de los inconvenientes, se suscitan dentro del sistema educativo.

En el 2013, se creó el INEVAL, mismo que tiene como ocupación causar la calidad educativa mediante el sistema educativo que se ejecute en el momento. Buscando en el estudiante mostrar los logros de aprendizaje como indicadores fundamentales para la calidad y desempeño educativo; pero esencialmente establecer los cimientos de desarrollo de un país.

Desde el año 2014 se presentan las pruebas ser bachiller en el Ecuador con el objetivo de determinar el nivel de conocimiento en las áreas básicas como Matemática, Lengua y Literatura, Ciencias Naturales, Ciencias Sociales, mismo que según el INEVAL (2018)

El examen Ser Bachiller aporta con el 30\% a la nota final para graduarse de bachiller, el promedio obtenido en el subnivel de básica superior, aporta con el $30 \%$ y el promedio de los tres años de bachillerato con el $40 \% "$ estas dos últimas corresponden a la nota final de graduación. De aquí que es imperante que el perfil de egreso de los estudiantes que cursan los últimos años de bachillerato se equipare a las directrices que se enmarcan en las pruebas Ser Bachiller (p.11).

Cada año, estudiantes del nivel secundario entiéndase como nivel secundario a colegio egresan y aspiran continuar su vida estudiantil en la educación superior, pero estas aspiraciones frecuentemente se ven estancadas por falencias en los conocimientos adquiridos anteriormente y el perfil educativo que la educación superior requiere. Es aquí donde se necesita emparejar las acciones que se presentan en los dos ámbitos y conocer cuánto influye el uno del otro.

Actualmente en Ecuador el INEVAL establece como logros alcanzados el nivel elemental, en cuanto al bachiller ecuatoriano, es decir solo cumple con las expectativas básicas. A futuro si la educación sigue de la misma manera no logrará formar bachilleres diferentes al que se forman actualmente. Y si las exigencias son mayores la educación decaería. Es por ello que el BGU por más pomposo que suene no va lograr una solución, la alternativa es mejorar aquello que estamos haciendo mal. Y que las expectativas del nivel educativo lleguen a niveles más competitivos. 
En concordancia este estudio busca conocer algunas aristas para determinar la relación del perfil de egreso de los estudiantes de Bachillerato General Unificado para el ingreso a la carrera de pedagogía en Ciencias Experimentales de la Informática de la Facultad de Filosofía Letras y Ciencias de la Educación en la Universidad Central del Ecuador. Para ello, se establecen los siguientes objetivos específicos:

- Diagnosticar las destrezas adquiridas por los estudiantes durante el bachillerato general unificado en las áreas que evalúa el programa ser bachiller.

- Determinar la relación entre destrezas del perfil de ingreso a los estudios superiores a la Carrera de Pedagogía en Informática y el perfil de egreso del Bachillerato General Unificado que permite acceder a la universidad.

- Establecer el nivel de conocimiento que poseen los estudiantes en las asignaturas del tronco común para ingresar a la carrera de pedagogía en informática.

Por consiguiente, el estudio está estructurado en el inicio de ilustraciones concernientes al bachillerato en sus aspectos básicos, además de experiencias formativas acerca del BGU, posterior a ello, se plantean conceptos y definiciones en relación al bachillerato general unificado. Consecuentemente se presenta la metodología utilizada durante el diseño de la investigación misma que contiene un enfoque cuantitativo de campo, con soporte documental y de nivel explicativo, seguido de resultados que se obtuvieron, $y$, finalmente se presentan las conclusiones.

\section{Fundamentación Teórica}

A criterio del Ministerio de Educación el perfil de salida se define a partir de tres valores fundamentales: la justicia, la innovación y la solidaridad y establece, en torno a ellos, un conjunto de capacidades y responsabilidades que los estudiantes han de ir adquiriendo en su tránsito por la educación obligatoria (...) escrito en primera persona del plural, pensando que los estudiantes se apropien de él y lo tomen como un referente en su trabajo cotidiano en el aula (Ministerio de Educación 2019). Por otro lado, el perfil de ingreso o cobertura es la capacidad de oferta que logra un sistema educativo de satisfacer toda la demanda social, en todos los grados escolares y en todos los espacios geográficos de un país. La cobertura relaciona el número de estudiantes matriculados con el número total de jóvenes en edad escolar y con opción de seguir los estudios de básica, secundaria y media.

En una investigación realizada en la Facultad Latinoamericana de Ciencias Sociales FLACSO Ecuador por parte de Santana (2015) se explican las causas por las que existen fallas de implementación en la política de educación en bachillerato en Ecuador, a partir de la LOEI 2011. El estudio se enfoca en las fallas políticas públicas, la falla de gobernanza, la falla del Estado principalmente. En este sentido establecen que es importante que el gobierno de turno enfoque su apoyo de manera prioritaria a la educación, y así establezca parámetros para mostrar mejoras en el sistema educativo. Entregar a la sociedad bachilleres con conocimientos adecuados a las necesidades de la sociedad, pero esto no se logrará si no se distribuye de manera igualitaria los fondos económicos y no solo enfocados a ciertos sectores.

Los resultados educacionales provienen de varios factores y son: la educación formal, la habilidad natural, los antecedentes y educación familiar, la situación de carencia, pobreza o exclusión. Los resultados de la educación formal se evidencian en la eficiencia y la equidad. La eficiencia es la capacidad del sistema educativo para promover estudiantes que ingresan a un nivel dentro de un plazo en una mayor proporción. La equidad está relacionada con la distribución de los resultados, es decir que una población pobre está asociada con peores resultados, y por tanto con peores ingresos. 
Al mencionar que el gobierno pretende homologar los conocimientos en los estudiantes ecuatorianos y así evitar esparcimiento de conocimientos, se presentan apreciaciones distintas a las esperadas. Mostrando así una desigualdad de metas alcanzadas y objetivos por alcanzar dispersos en el tiempo incluso en el espacio. En la actualidad los docentes no se centran en el estudiante y lo que enseña, más el trabajo del docente tiene mayor carga administrativa haciendo que no se centre en el estudiante.

El objetivo del gobierno es alcanzar conocimientos uniformes, pero aquello no se ha podido reflejar en los bachilleres de Ecuador. Aún más es difícil creer que el bachiller del oriente ecuatoriano tiene las mismas necesidades que el de la ciudad. Las realidades de cada uno de ellos son diferentes, pues las necesidades y oportunidades cambian a diferencia del lugar en donde se ubican. Como conclusión en la investigación citada se pretende plasmar el entorno que engloba a la educación y particularmente al bachillerato general unificado y su aporte a los estudiantes en su traslado a la educación superior, determinando asimetría al querer agregar importancia en lo político y en lo programado haciéndolo difuso y ambivalente afectando esto al sistema directamente. Los estados al encontrarse inmerso en la figura de encaminamiento a las mejoras educativas no plasman de manera adecuada el modelo de gestión desconcentrada y así disminuye el valor que presentan los involucrados en ello, como son los docentes, autoridades educativas incluso los mismos estudiantes.

Esto permite concluir que el sistema educativo planteado no responde a los perfiles de salida que se proyecta para los bachilleres del Ecuador. Estas afirmaciones aportaron para realizar mejoras en el currículo presentado en el 2016, también evidenciando que es preciso tomar en consideración lo que cataloga enfáticamente uno de los directores de las pruebas PISA que son acertadas pues el inconveniente mayoritario que tiene Latinoamérica dentro de la educación se centra en la reforma curricular y son netamente de diseño. Pues se debe realizar las reformas educativas sobre las realidades que existen en el país, y no enfocados en realidades ajenas.

En educación se discute mucho sobre las mejoras tanto en calidad como en infraestructura, es por ello que el Sistema de Información de Tendencias Educativas en América Latina (SITEAL) en el documento extraído de la Base de normativas y políticas sobre el Nuevo Bachillerato Ecuatoriano del Ministerio de Educación actualizada a diciembre 12 del 2018, cuyo objetivo es la reestructuración de las reformas curriculares diseñadas en el año 2008 enfoca una revisión profunda en la reforma del 2010 para mejorar la educación en el Ecuador con énfasis en el segundo nivel de educación como lo es el Bachillerato. A criterio de la investigación es allí donde se debe realizar reformas concretas, centrarse en lo primordial como el estudiante y las necesidades que tiene la sociedad en la actualidad. Mejoras que se presentarán cuando el ciudadano ecuatoriano termina el bachillerato, y las diferentes vías que deben presentarse al egresar del mismo. Es decir que, el bachiller ecuatoriano debe enfocarse en seguir una carrera de educación superior, pero sí por motivos ajenos debe enrolarse en el ámbito laboral, logre hacerlo sin ningún inconveniente.

A criterio de SITEAL 2018, para desempeñar las funciones formativas, capacitadoras y propedéuticas, el currículo del bachillerato debe estar bien articulado con las demandas laborales de los diferentes sectores productivos, de las universidades y otros centros de educación superior, de tal manera que no haya una ruptura excesiva entre uno y otro. Asimismo, es necesario que el bachiller interesado en ingresar a la universidad no se vea limitado en sus opciones como resultado, justamente, de una especialización prematura y exageradamente parcelada, para lo cual es imperioso que su formación haya sido lo suficientemente genérica como para que ninguna opción de estudios superiores quede excluida (p. 10).

Licencia Creative Commons Atribución 4.0 Internacional (CC BY 4.0)

Revista Cátedra, 5(1), pp. 119-130, enero-junio 2022. e-ISSN: 2631-2875

https://doi.org/10.29166/catedra.v5i1.3428 
El sistema educativo pretende responder a las necesidades de la sociedad actual, y así subsanar los problemas que el bachillerato presenta. Principalmente uno de los inconvenientes son los distintos enfoques que presenta el currículo en el sistema educativo exponiendo una dispersión frente a lo que se propone; partiendo del enfoque "de competencias" versus el "enfoque de contenidos". Si se pretende una homologación en educación los enfoques deberían ser el mismo.

Los problemas exteriorizados en los currículos del 2008, y en la actualización y fortalecimiento del currículo de marzo del 2010 mostraron necesidades que los estudiantes en el Ecuador enfrentaban posterior al egresar del bachillerato problemas tales como desigualdades socioeconómicas, falta en las garantías de igualdad de oportunidades, dualismos formativos entre otras. Se han presentado innumerables reuniones, debates, congresos, charlas, pero a decir verdad los frutos que estas han generado han sido poco fructíferas; en las mejoras que la educación debe tener. Estos inconvenientes no son efectos que se concentran en la educación media, sino que se lo arrastra hasta la educación superior, es por ello que se replantea una nueva reforma, estas no serían inéditas ya que existen debates constantes en América latina sobre una educación por "generalidades" y "especialidades".

\section{Metodología}

A continuación, se describe de manera general la metodología utilizada durante la investigación, para lo cual se determina el diseño de la investigación, población, muestra y las técnicas e instrumentos de investigación.

\subsection{Diseño de investigación}

La investigación realizada responde a un estudio cuantitativo, con diseño no experimental trasversal y alcance explicativo cuya intención fue establecer el aporte del bachillerato general unificado en los estudiantes, que pretendían ingresar a la educación superior dentro de la Facultad de Filosofía Letras y Ciencias de la Educación de la Carrera en Pedagogía en Informática en los estudiantes de nivelación en el periodo octubre 2019-marzo 2020.

A continuación, en el cuadro 1, se describen las principales variables del estudio:

\begin{tabular}{llll}
\hline \multicolumn{1}{c}{ Variable } & Dimensión & Indicadores & Ítems \\
\hline & Ámbito cognitivo & $\begin{array}{l}\text { Razonamiento lógico } \\
\text { Matemática }\end{array}$ & $1.1,1.2,1.3,1.4,1.5,1.6$ \\
& & Lenguaje & \\
El perfil de egreso & & Entorno social & $1.7,1.8,1.9$ \\
de los estudiantes & & Ciencias naturales & $1.10,1.11,1.12$ \\
de bachillerato & Ámbito & Selecciona & $1.13,1.20$ \\
general unificado & procedimental & Analiza & $2.14,2.15$, \\
& & Completa & $2.16,2.17$, \\
& & Jerarquiza & $2.18,2.19$, \\
& Ámbito axiológico & Respeto & \\
& Saber ser & Solidaridad & $3.21,3.22,3.23$ \\
& & Honestidad & 3.24 \\
& & & $3.25,3.26$
\end{tabular}




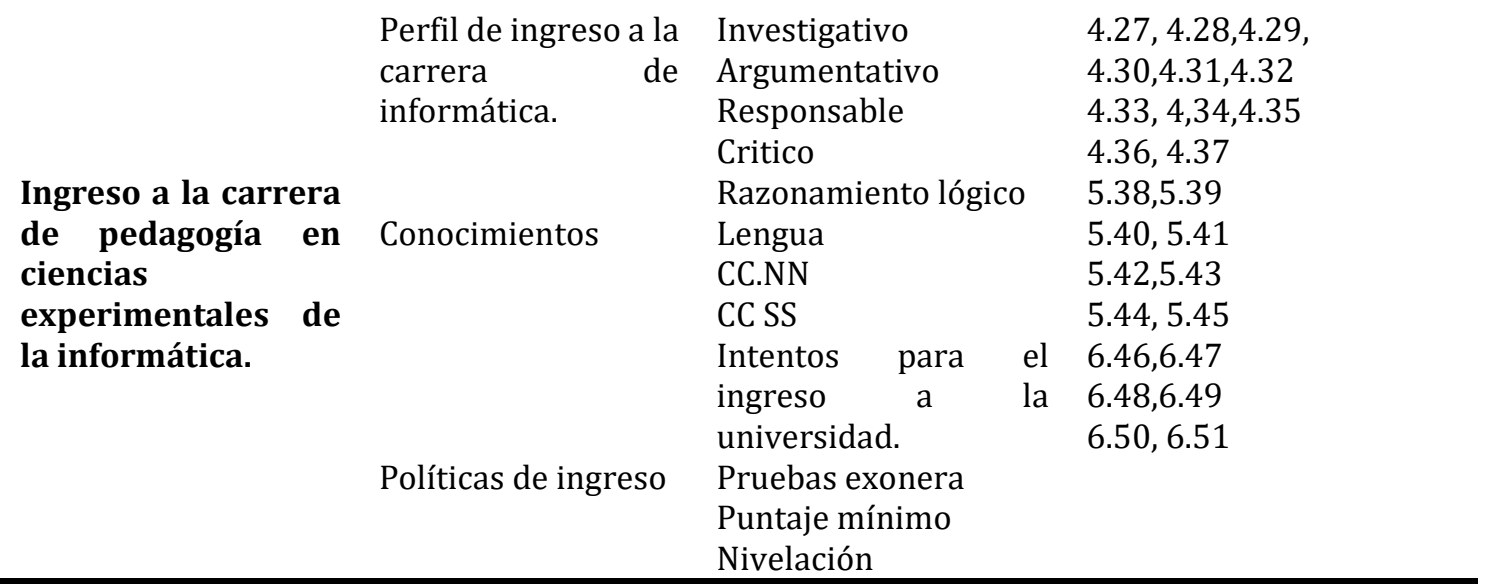

Cuadro 1. Operacionalización de las variables estudiadas

\subsection{Población y muestra}

La población estuvo constituida por 51 estudiantes que asisten a las jornadas matutina y vespertina de la Facultad de Filosofía Letras y Ciencias de la Educación de la Universidad Central del Ecuador, de los cuales el $60 \%$ de género masculino, y el $40 \%$ restante femenino. Según la edad, el 76\% se encontraban entre los 20 y 21 años, y el 52\% de los estudiantes formó parte del programa del bachillerato general unificado.

\subsection{Técnicas e instrumentos de investigación}

Para la recolección de los datos referidos a las variables descritas, se utilizó una encuesta en forma de cuestionario con preguntas medidas en una escala de likert y diseñado bajo procedimientos especificos propios de la disciplina en la investigación. El instrumento constó de 51 preguntas, divididos por constructos de dominio lógico, linguistico, cientifico, axiológico, cada constructo englobó tres preguntas adaptadas a la escala de Likert en un rango de 5 a 1 donde 5 es el rango más alto.

El instrumento fue sometido previamente a un análisis de confiabilidad para lo cual se utilizó una prueba piloto con 10 estudiantes de la Carrera de Pedagogía en Ciencias Experimentales de la Informática sección matutina, con características similares a la muestra objeto de investigación. La prueba piloto sirvió para destacar los errores que se presentaban en el instrumento mismo que se realizó con una encuesta de carácter presencial; realizados los cambios pertinentes posteriormente se aplicó a la población en su totalidad. En este sentido, se obtuvo un coeficiente de concordancia interna de los items alfa de cronbach de 0,831 , lo cual representa una alta confiabilidad.

\section{Resultados}

De forma descriptiva se tiene una muestra compuesta por un $40 \%$ de mujeres y $60 \%$ de hombres, con un $48 \%$ en el grupo de edad entre los 20 a 21 años, seguido por un $28 \%$ en edades de 18 a 19 años, finalmente el $24 \%$ de los estudiantes oscilan en edades entre 22 a 23años de edad. En la Carrera de Pedagogía de las Ciencias Experimentales de Informática el $52 \%$ de sus estudiantes tiene como título de nivel secundario el bachillerato general unificado, seguido por el $44 \%$ de los estudiantes con el bachillerato técnico, y un $4 \%$ el bachillerato por especialidades u otra. Del grupo encuestado 26 estudiantes de los 52 egresaron del nivel secundario con bachillerato general unificado, también 22 estudiantes obtuvieron el bachillerato técnico. Y 4 estudiantes obtuvieron otro tipo de bachillerato. De allí que se presenta un resumen de los resultados obtenidos por las dimensiones de cada una de las variables objetivos.

Perfil de egreso de los estudiantes de bachillerato general unificado

Licencia Creative Commons Atribución 4.0 Internacional (CC BY 4.0) 
Dimensión ámbito cognitivo en referencia al perfil de egreso de los estudiantes del BGU en el ámbito cognitivo muestran falencias en el razonamiento lógico y matemático, ya que la mitad más uno contestó de forma incorrecta y el cuarenta por ciento restantes correctos, los demás no respondieron. El dominio lingüístico en los estudiantes es mejor en la mitad más uno mostrando habilidades adecuadas en el manejo de la literatura. Además, menos de la cuarta parte de los estudiantes domina adecuadamente la asignatura de estudios sociales. Mismo que permite evidenciar un $48 \%$ de respuestas acertadas en promedio. Dados los valores se ha evidenciado grandes debilidades cognitivas tanto en los indicadores de razonamiento lógico, matemático, social y natural en los estudiantes participantes del programa de bachillerato general unificado.

Dimensión ámbito procedimental para conocer si las habilidades superiores fueron adquiridas por el estudiante, se realizó el proceso correspondiente mismo que reflejó que en promedio 38\% de los estudiantes adquirió las habilidades superiores. Además, al consultarles a los estudiantes el dominio cognitivo en conjunto con el procedimental se evidencio que menos de la mitad de los encuestados respondió adecuadamente a las preguntas cuando estas se mostraron en conjunto. Durante este proceso existe superioridad en cuanto los elementos dentro de los temas que abarco la encuesta, razón por la cual al agrupar las dimensiones ámbito cognitivo con el procedimental los resultados fueron inferiores a la mitad menos dos.

Dimensión ámbito axiológico con relación a las determinaciones axiológicas en el estudiante al respecto, los encuestados se mantienen de acuerdo a los ítems formulados, en cuanto honestidad mantienen posturas en la escala de acuerdo y en cuanto a solidaridad los estudiantes encuestados frecuentemente se encuentran muy de acuerdo. Es relevante mencionar que las prácticas dentro del ámbito axiológico se reflejan dentro del rango de buenas sin llegar al nivel de muy buenas sobre las perspectivas que se tiene en este aspecto.

Ingreso a la Carrera de Pedagogía en Ciencias Experimentales de la Informática en el ámbito investigativo la mitad menos uno contesto que investiga autónomamente, además la cuarta parte menos uno investiga cuando no conoce sobre un tema, también el estudiante considera que es argumentativo casi siempre en un 34\%. El nivel de responsabilidad del estudiante fue tan solo de un $28 \%$ que siempre muestra responsabilidad acorde a las expectativas de la carrera. En cuanto si el estudiante es crítico durante una clase los informantes respondieron que a veces son críticos en un 38\% durante una clase.

En la dimensión Conocimientos las asignaturas abarcadas dentro de la dimensión de conocimientos fueron las mismas contenidas dentro del ámbito cognitivo, aspectos similares que se miden dentro de las evaluaciones de las pruebas ser bachiller. El promedio en las respuestas correctas alcanzó solamente el 10,25\%, evidenciando que la mayoría de los estudiantes encuestados posee grandes debilidades en cuanto a los conocimientos adquiridos.

En cuanto la dimensión Políticas de ingreso las políticas de ingreso generan descontento en los estudiantes, debido a que la gran mayoría ha intentado más de una vez alcanzar el puntaje requerido para optar por el ingreso a la educación superior y otros no recuerdan las veces que lo han intentado. El aporte mínimo que el programa de bachillerato general unificado representa hace que la totalidad de los estudiantes tenga que tomar el semestre de nivelación antes de ir al primer semestre de carrera. En teoría la nivelación debería ser de utilidad para iniciar con éxito la carrera sin embargo más de la cuarta parte de los informantes opina que el periodo de nivelación no representa una ayuda adecuada. En promedio un $40 \%$ de los estudiantes considera que la prueba exonera no ayuda a pasar el semestre de nivelación ya que abarca temas con un alto nivel de complejidad. Para la correlación general se determinó mediante la variable uno o independiente el perfil de 
egreso de los estudiantes de bachillerato general unificado; y la variable dos o dependiente perfil de ingreso.

Con respecto al ingreso a la Carrera de Pedagogía en Ciencias Experimentales de la Informática en función del perfil de egreso del BGU, se determinó que existe correlación inversa poco significativa entre las variables con un valor de -0,136. Esto conlleva a aceptar la hipótesis dos misma que recae en la hipótesis nula (H0) El perfil de egreso de los estudiantes de bachillerato general unificado no aporta las bases suficientes para ingresar a la educación superior. Además de la correlación general se analizó las dimensiones de la operacionalización de las variables ámbito procedimental con el perfil de ingreso para conocer si existe relación con los indicadores. En concordancia, el coeficiente de correlación entre el perfil de ingreso de la carrera de pedagogía en informática, en el ámbito procedimental con el perfil de egreso de los estudiantes del bachillerato general unificado fue de 0,986. Evidenciando un alto nivel de correlación afirmando las necesidades de la educación superior en cuanto el ámbito procedimental del bachiller, sobre las destrezas que debe presentar y su capacidad de seleccionar, analizar, y hasta jerarquizar; habilidades adquiridas por el estudiante durante su paso por la secundaria, son altas y constituyen una ventaja para el ingreso a la carrera.

Con este resultado se comprueba que lo aceptable del BGU son las relaciones que existen entre Preingreso y el ámbito procedimental aceptando la hipótesis de que las destrezas adquiridas en el bachillerato general unificado no son elementales, y aportan al perfil de ingreso a la educación superior.

\section{Conclusiones}

La investigación en esencia buscó conocer la relación entre el perfil de egreso del bachillerato general unificado y el perfil de ingreso a la carrera de informática misma que tuvo como población principal a los estudiantes de la carrera de primer semestre.

En relación al objetivo específico uno que buscó diagnosticar las destrezas adquiridas por los estudiantes durante el BGU se logró determinar que el 52\% de los estudiantes tienen falencias en el ámbito cognitivo como son razonamiento lógico, matemático, estudios sociales y ciencias naturales. Es necesario mencionar que la única fortaleza se evidencio en el área de dominio lingüístico con un $60 \%$ de asertividad

Esto contrasta con Tamayo (2019) en su investigación denominada "Sistema Educativo de Ecuador: un sistema, dos mundos" donde menciona que si las pruebas eran complicadas en 2008 cuando 4 de cada 10 estudiantes no pasaron las pruebas de ingreso(..) "Esto se agravó más con el cambio de las pruebas desde el razonamiento abstracto, numérico y verbal de la primera aplicación del examen en 2012 (SENESCYT, 2015) a su versión actual como examen de conocimientos.

Con relación al objetivo específico dos para determinar la relación entre destrezas del perfil de ingreso a los estudios superiores y el perfil de egreso del Bachillerato general unificado que permite acceder a la universidad. Se concluyó que las destrezas desarrolladas durante el BGU apenas alcanzan el nivel de criticidad que se requiere, también se evidencia el bajo nivel de responsabilidad y argumentatividad, es decir las expectativas del perfil de ingreso son altas y las destrezas desarrolladas son bajas.

En comparación con Ortiz (2017), durante su investigación: “Vivencias en la Universidad Central del Ecuador" (p. 50) determinaron que, en la FJCPS, la capacidad de pensar en modo crítico tuvo como resultados a 44\%, 44\%, y 44\% durante tres períodos. En la FAU, la capacidad de pensar en modo crítico tuvo como resultados a 33\%, 50\%, y 58\% en los tres períodos es importante mencionar que los resultados que se muestran son resultados 
obtenidos en facultades diferentes, obtenidas en el curso de nivelación del año 2012 al 2015. (p. 48).

En relación al objetivo específico tres, establecer el nivel de conocimiento que poseen los estudiantes en las asignaturas del tronco común para ingresar a la Carrera de Pedagogía en Informática. Se encontró que nivel del conocimiento en las áreas de razonamiento lógico, matemáticas, lengua, estudios sociales y ciencias naturales es bajo. En relación a las altas expectativas que el ministerio de educación afirmó se alcanzaría con la incorporación del Bachillerato general unificado al sistema educativo.

En lo concerniente a la investigación de los autores antes mencionados sobre establecer el nivel de conocimiento que poseen los estudiantes. En las asignaturas del tronco común para el ingreso a la carrera se evidencia que el razonamiento lógico no es una asignatura alcanzada adecuadamente. y otras que se presentaron dentro de la investigación que concluye que se debe reforzar en desarrollo del pensamiento y razonamiento lógico, en determinadas facultades de las estudiadas habilidades orales y escritas además del razonamiento verbal. Donde también se evidencia porcentajes altos en respuestas acertadas en ciertos periodos y bajos en otros. 


\section{Bibliografía}

Instituto de Evaluación Educativa. (2019). Informe de resultados nacional. http://www.evaluacion.gob.ec/evaluaciones/investigaciones-ser-bachiller/.

Ministerio de Educación del Ecuador. (0 de 0 de 2019). Currículo de los niveles de educación obligatoria. Recuperado el 21 de 3 de 2021, de Ministerio de Educación Currículo: https://educacion.gob.ec/curriculo/.

Santana, C. I. (04 de 01 de 2016). La reforma del Bachillerato en el Ecuador entre 20072014: caso de la Unidad Educativa Fiscal 13 de octubre de Calceta-Manabí. Recuperado el 24 de 06 de 2019.

Ortiz Herrera, J. D., Buitron Aguas, L. H., \& Yupangui Carrillo, Y. de las M. (2017). Nivelación propuesta por la SENESCYT: Vivencias en la Universidad Central del Ecuador. INNOVA $\begin{array}{lll}\text { Research } \quad \text { Journal, 2(10.1), } & \text { 42-53. }\end{array}$ https://doi.org/10.33890/innova.v2.n10.1.2017.491

Bachillerato General Unificado. (s. f.). Ministerio de Educación. Recuperado 10 de febrero de 2021, de https://educacion.gob.ec/bachillerato-general-unificado/

Instituto Nacional de Evaluación Educativa. (10 de 06 de 2013). Resultados Educativos INEVAL. Recuperado el 22 de 03 de 2020, de https://www.evaluacion.gob.ec/inevalpresento-resultados-educativos-2/

Reglamento general a la ley orgánica de educación intercultural, 116 (2012). https://educacion.gob.ec/wp-content/uploads/downloads/2017/02/Reglamento-

General-a-la-Ley-OrgAnica-de-Educacion-Intercultural.pdf

Santana Cobo, M. C. (2015). Análisis de las fallas de implementación de la política de educación para bachillerato en Ecuador, a partir de la reforma educativa de 2011 [Facultad Latinoamericana de Ciencias Sociales-Flacso Ecuador]. http://hdl.handle.net/10469/13597

Madrid-Tamayo, T. (2019). El sistema educativo de Ecuador: un sistema, dos mundos. Revista Andina de Educación, 2(1), 8-17. https://doi.org/10.32719/26312816.2019.2.1.2

Rosero, M. (2020, enero 26). Lorena Araujo: 'La "U" debe reflexionar sobre qué necesita el país' | El Comercio. El Comercio, 2. https://www.elcomercio.com/actualidad/lorenaaraujo-universidad-educacion-bachiller.html.

\section{Autores}

GLADYS CABASCANGO-TRÁVEZ Obtuvo su título de Magister en Educación con mención Gestión del aprendizaje mediado por TIC en la Universidad Central del Ecuador, obtuvo su título de Licenciada en Ciencias de la Educación mención Informática en la Universidad Central del Ecuador.

Actualmente es docente de Educación Superior y bachillerato. Ha sido tutora de tesis y proyectos enfocados en aprendizajes basados en proyectos.

HAMILTON PEREZ-NARVÁEZ obtuvo su título de Licenciado en Ciencias de la Educación especialización Informática, Especialista en Entornos Virtuales OEI. Magíster en Educación Superior y Doctor en Investigación Educativa en la Universidad de Alicante. Ha escrito varios artículos relacionados con la Tecnología Educativa y el pensamiento computacional. 
Profesor de Bachillerato Técnico en Informática, Profesor de la Universidad Central del Ecuador en las cátedras de: Programación, Didáctica de la Informática y Pedagogía, profesor de la Pontificia Universidad Católica del Ecuador en cursos de TIC.

Actualmente es director de la carrera de Pedagogía de las Ciencias Experimentales en Informática y coordinador de la Red ecuatoriana de tecnología e innovación educativa. Ha sido tutor de tesis de grado y posgrado en la Facultad de Filosofía, coordinador de Maestría en Educación mención Gestión del aprendizaje mediado por TIC.

JAVIER GUAÑA-MOYA Recibió el Doctor of Philosophy in Computer Science, Master Degree in Distance Education Elearning, Maestría en Conectividad y Redes de Telecomunicaciones, Master en Educación, Especialista en Currículo y Didáctica y el Diplomado en Investigación Socioeducativa. Sus estudios de tercer nivel son Ingeniero en Electrónica y Telemática, Ingeniero en Sistemas de Información. Catedrático de Postgrado de la Pontificia Universidad Católica del Ecuador (PUCE), Universidad Técnica de Ambato (UTA), Universidad Católica de Cuenca (UCC), Universidad Tecnológica Empresarial de Guayaquil (UTEG), Universidad Israel y de la Universidad Luis Vargas Torres.

Actualmente es catedrático de Pregrado en la Pontifica Universidad Católica del Ecuador, Universidad Central del Ecuador (UCE), Universidad de las Américas (UDLA), Universidad Metropolitana del Ecuador (UMET), Universidad Tecnológica América.

NELSON SALGADO-REYES graduado como Ingeniero Informático en 1999 por la Universidad Central del Ecuador, Magister en Dirección y Administración de Empresas por la Universidad Tecnológica Israel, Magister en Docencia e Investigación Universitaria por la Universidad Nacional de Loja y Doctor dentro del Programa de Tecnologías Informáticas y Comunicacionales (TINC) 2017, por la Universidad de Extremadura España.

Desde 2017 hasta la fecha trabaja como docente principal en la Pontificia Universidad Católica del Ecuador, en la Universidad Central del Ecuador, especialidad Bases de Datos, Las líneas de interés: modelamiento de datos, calidad de software, desarrollo de aplicaciones, docente de programas de maestría en varias universidades públicas y privadas del Ecuador. 